\title{
Correction to: Interpretable and Annotation-Efficient Learning for Medical Image Computing
}

\author{
Jaime Cardoso (1D, Hien Van Nguyen (D), Nicholas Heller (D), \\ Pedro Henriques Abreu (D), Ivana Isgum (D), Wilson Silva (D), \\ Ricardo Cruz (D), Jose Pereira Amorim (D), Vishal Patel, \\ Badri Roysam, Kevin Zhou, Steve Jiang, Ngan Le, Khoa Luu, \\ Raphael Sznitman (D), Veronika Cheplygina, Diana Mateus (D), \\ Emanuele Trucco (D), and Samaneh Abbasi (D)
}

\section{Correction to: \\ J. Cardoso et al. (Eds.): Interpretable and Annotation-Efficient Learning for Medical Image Computing, LNCS 12446, https://doi.org/10.1007/978-3-030-61166-8}

The original version of this book was revised. The following corrections were implemented:

The acronym was corrected to "MIL3ID" throughout the book.

The equation on page 5 of Chapter 14 was modified to improve its accuracy and readability. 